\title{
Below the Standard Model?
}

\author{
Jacques Consiglio $^{1}$ \\ ${ }^{1}$ Labastidette, France \\ Correspondence: Jacques Consiglio, 52, Chemin de Labarthe, F31600 Labastidette, France. Tel: 33-687-493-664. \\ E-mail: Jacques.Consiglio@gmail.com
}

Received: January 24, 2014 Accepted: February 10, 2014 Online Published: February 20, 2014

doi:10.5539/apr.v6n2p19

URL: http://dx.doi.org/10.5539/apr.v6n2p19

\begin{abstract}
In two previous papers, we showed that that the observed elementary particles masses spectrum agrees with a single resonance equation. Using those results we show that the resonance numbers address three distinct resonance schemes - one for each symmetry $\mathrm{U}(1)$ and $\mathrm{SU}(3)$, and one for massive bosons; we then deduce, from the equation parameters, a unique fundamental field "below" the standard model fields and forces, which we interpret as electric and magnetic poles and magnetic currents.
\end{abstract}

Keywords: mass spectrum, standard model, forces unification

\section{Introduction}

\subsection{Context}

The standard model (SM) of particles physics is based on three fundamental symmetries and conservation laws, namely $\mathrm{SU}(3) \mathrm{xU}(2) \mathrm{x}(1)$, quantum numbers, energy and momentum, and a scalar field. Current theoretical research digs beyond and expects new particles, or their absence, to verify or rule out new theories; an important motivation to this search is the so called absence of naturalness. In particular the elementary particles mass spectrum looks quite random and SM provides no solution to its origin.

In this paper we start from phenomenology. The analysis is based on a resonance equation that was shown in agreement with the estimated mass of all known particles, including the Higgs boson. We will show that the resonance numbers are natural with respect to geometry and charges, and that a unique field is there. We interpret the field as made of two and only two types of charges and currents: electric and magnetic.

\subsection{Methodology}

In two previous papers (Consiglio, 2012a, 2012b), we showed that the following resonance equation matches all known and estimated masses (i.e. pole masses coming in the SM Lagrangians):

$$
m=X /(K D+1 / N P)^{3}+\mu
$$

Where:

- $\quad X$ is a density (assumed universal).

- $\quad K, N$, and $P$ are small integral numbers (or a fraction in one case).

- $\quad D$ is a distance specific of the particle group (electrons, quark, or massive boson).

- $\quad \mu$ is a small "naked mass" specific of the particle group (that we compute where possible).

The initial model leading to (1) is an absorber theory based on a resonance driven absorption/emission process and the existence of a pressure field $X$, where particles masses correspond to an exclusion or depletion volume of radius $(K D+1 / N P)$. Essentially, a single fundamental resonance exists $(K=0, N=P=1)$ and particles occupy specific double-harmonics $N P$. The $K D$ shifts the mass in a particle-dependent way; $D$ is specific to each group of particles (electrons, quarks, and massive bosons) while $N, P$, and $K$ are particle-dependent. The trick is that $K D$ is assumed quantized, and we shall see that it holds quite well and gives interesting insights.

On this basis it is legitimate to work a link with the SM Higgs field, and hopefully the weak and strong forces; but the problem is that with Equation (1) we are almost blind. It contains no information related to different symmetries or charges; we do not even know what kind of space it addresses. However, consider that $K D$ is quantized, and unit-less, and depend on the particles group to which (1) is applied. Its quantization should relate to a potential where we should find known coupling constants. 
We will then search for formal coincidences with the values of these parameters $(D$ and $\mu)$. We naturally expect some simple expression in $\alpha$ for electrons, $\alpha_{S}$ for quarks... We also expect random coincidences and we must avoid their use in the reasoning; the method requires caution.

Similarly, the resonance numbers $N, P$ and $K$ cannot be random; they must depend on the resonance structure and, since the resonance is double and shown group-dependent, it is legitimate to use the results (specific numbers $N, P, K$ and parameters) to deduce some information related to the resonances geometry.

\section{Particles Mass, Tracking Coincidences}

\subsection{Electrons}

Table 1 shows the numbers we found for the electron, muon, and tau. The search was not systematic (manual), and then not exhaustive; but the numbers we found $(N, P, K$, and mass $\mu)$ are minimal.

Table 1. Electrons, muon, tau. * $\mathrm{MeV} / \mathrm{c}^{2}$

\begin{tabular}{lcccll}
\hline Particle & $\mathrm{P}$ & $\mathrm{N}$ & $\mathrm{K}$ & Computed $(*)$ & Measured $(*)$ \\
\hline Electron & 2 & 2 & 2 & 0.510998928 & $0.510998928(11)$ \\
Muon & 5 & 5 & 3 & 105.6583715 & $105.6583715(35)$ \\
Tau & 9 & 9 & 5 & 1776.84 & $1776.82(16)$ \\
\hline
\end{tabular}

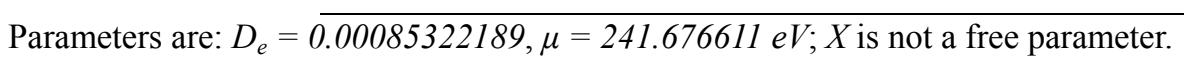

We first notice that the $N, P, K$ are quite small, and obey a regular pattern $(N=P$, and $K$ successive primes); the result in Table 1 is not far-fetched - it looks naturally plausible. The values of $K$ are all positive; in a spatial representation of resonances, it corresponds to a repulsive force that we assume related to the electric field.

Equation (1) comes from an absorber theory and then we should check our results with respect to quantum electrodynamics (QED). Bethe (1947) computes the hydrogen Lamb shift; he gets:

$$
\Delta E=\left(\alpha^{5} m_{e} c^{2} / 6 \pi\right) \log \left(m_{e}{ }^{2} c^{2} / 8.9 \alpha^{2} m_{e}{ }^{2} c^{2}\right)
$$

where $m_{e}$ is the electron mass; the expression in the logarithm depends on the cutoff and gives a ratio between the electron's self-interaction and absorption, which, in our case, should be $\mu$ and $\left(m_{e}-\mu\right)$ respectively; we find:

$$
\left(m_{e}-\mu\right) / \mu=1 / 8.8857 \alpha^{2}
$$

The relative difference with respect to Bethe's result is $1.6 \cdot 10^{-3}$ (or $2 \cdot 10^{-4}$ for $\Delta E$ ) and then $\mu$ is relevant.

We then notice a first coincidence:

$$
\left(m_{e}-\mu\right) / \mu=\sqrt{ } 2 / 4 \pi \alpha^{2}
$$

The relative difference in (3) with respect to $\sqrt{ } 2$ is $\approx 1.25 \cdot 10^{-5}$, it gives a difference in mass $\Delta \mu \approx 3 \mathrm{meV}$, which is well within uncertainty of the electron mass precision $( \pm 11 \mathrm{meV})$. The factor $4 \pi$ can be related to dispersion and the $\sqrt{ } 2$ can come from a squared mass or from geometry. The coincidence could then relate to the absorber scheme (See also addendum 2, a conceptual correspondence with QED, and an analysis consistent with (3)).

Since we only analyze ratios of mass and distance, $D_{e}$ is unit-less; a second coincidence is:

$$
D_{e}=16.0225 \alpha^{2}
$$

Surprisingly, (4) is also a coincidence in $\alpha_{S}$, the strong force coupling constant. This is a little shocking since electrons are not sensitive to the strong force. Now $\alpha_{S}$ is running and not known with very high precision, then we will use $\alpha_{S}=0.11692$; we get:

$$
D_{e}=\alpha_{S} \alpha
$$

\subsection{Quarks}

Table 2 shows the numbers we found for quarks.

The density $X$ is unchanged with respect to electrons; all masses are deduced from the electron mass using resonances but require a minor adjustment of $D$. The adjustment is necessary to fit the top quark mass range, since using $D_{q}=D_{e}$ gives $m_{t} \approx 162 \mathrm{GeV} / \mathrm{c}^{2}$. 
Table 2. Quarks resonances. (*) $\mathrm{MeV} / \mathrm{c}^{2}$

\begin{tabular}{llllllll}
\hline Particle & Charge & $\mathrm{P}$ & $\mathrm{N}$ & $\mathrm{K}$ & Computed $(*)$ & $\operatorname{Min}(*)$ & $\operatorname{Max}(*)$ \\
\hline Up & $2 \mathrm{e} / 3$ & 3 & $14 / 7$ & -6 & 1.93 & 1.7 & 3.1 \\
Down & $\mathrm{e} / 3$ & 3 & $19 / 7$ & -6 & 5.00 & 4.1 & 5.7 \\
Strange & $\mathrm{e} / 3$ & 3 & 7 & -6 & 106.4 & 80 & 130 \\
Charm & $2 \mathrm{e} / 3$ & 3 & 14 & -6 & 1,255 & 1180 & 1340 \\
Bottom & $\mathrm{e} / 3$ & 3 & 19 & -6 & 4,285 & 4130 & 4370 \\
Top & $2 \mathrm{e} / 3$ & 3 & 38 & -6 & 172,380 & 171700 & 173300
\end{tabular}

Parameters: $D_{q}=D_{e}(1+\alpha)=\alpha_{S} \alpha(1+\alpha)$; and $\mu$ cannot be found with current quarks' mass precision.

In Table 2, we remark:

- $\quad$ Only $N$ varies in Table 2; all NPs are harmonics of the same resonance $(P=3)$.

- $\quad$ All quarks with charge $2 / 3$ have $N$ even; all quarks with charge $1 / 3$ have $N$ odd or odd fractional (19/7).

- In generations 2 and 3, quarks have a ratio of 2 between $N s$, but the first generation is different.

- We use a fraction $(N=19 / 7)$ for the down; it gives a well centered mass and consistent harmonics.

- $\quad$ We get a nice harmonic progression in $N$ with ratios 2, 7, and 19.

Actually, quarks do not fit with mass series organized by generation, but by resonance + electric charge; it implies a direct connection between electricity and resonance and then between electricity and mass.

Starting with the up, the harmonic progression in $N$ is:

- $N(u) \times 7 \rightarrow N(c) \quad$ (same charges).

- $\quad N(c) \times 1 / 2 \rightarrow N(s) \quad$ (charge and resonance divided by 2 ).

Starting with the down:

- $\quad N(d) \times 7 \rightarrow N(b) \quad$ (same charges).

- $\quad N(b) \times 2 \rightarrow N(t) \quad$ (charge and resonance multiplied by 2 ).

Then a ratio 7 in $N$ conserves the charge while a ratio 2 changes it; the connection is obvious.

In Table 2, $K$ is a constant negative number; it shows (if compared to electrons) that an attractive force is also at work that reduces the exclusion volume, but the repulsive force is still there since quark masses are not infinite. The constancy of $K$ also shows that (1) cannot be changed (or tricked) to eliminate $D$, which is then significant. The shift in mass related to $K D_{q}$ is not negligible if compared to the resonance; it ranges from $1 / 6 / 6 D \approx 32.32$ for the up quark, to $1 / 38 / 6 D \approx 5.10$ for the top quark.

We find a coincidence quite similar to (3) (and already shown in Table 2 parameters):

$$
D_{q}=D_{e}(1+\alpha)=\alpha_{S} \alpha(1+\alpha)
$$

Then using quarks electric charge $( \pm 1 / 3, \pm 2 / 3$ ) (and still assuming that the repulsive force in electrons is due to their electric charge,) the forces at work in quarks and electrons are possibly related to a magnetic current, and quarks hold a magnetic charge that is roughly opposite to the Dirac condition. Assume that the repulsive force coefficient for electrons is a product $k e g$, it will give $k e g / 3$ or $2 k e g / 3$ for quarks. In the expression $k e g$, the coefficient $k$ is the cosine of the vertex angle of the Poincare cone, and then $k<1$. Then assuming quarks have a magnetic charge $g_{q}$, since the difference between $D_{q}$ and $D_{e}$ in $(5-6)$ is $1 \rightarrow(1+\alpha)$, the $\alpha$ comes from the quark magnetic charge and implies $\alpha k$ e $g / 3 \approx g_{q} g / 3$. Hence $g_{q}$ is roughly bounded by $\left|g_{q}\right|<2 \alpha e / 3$.

\subsection{Massive Bosons}

SM considers that the $\mathrm{W}^{ \pm}, \mathrm{Z}^{0}$ and $\mathrm{H}^{0}$ acquire their masses from a unique potential minimum which, in our logic, should correspond to the same resonance. This is what we find in Table 3 (Note that we use the ATLAS average estimate of the $\mathrm{H}^{0}$ mass; however we find a better fit with ATLAS measurement in the $\gamma \gamma$ channel $-126.5 \mathrm{GeV} / \mathrm{c}^{2}$ ).

The numbers in Table 3 fit with SM and a single potential minimum acting in the same manner on the $\mathrm{W}^{ \pm}, \mathrm{Z}^{0}$ and $\mathrm{H}^{0}$, because there is a single main resonance given by $N P=144$; but the mass is shifted for each particle. The shift is not so small if compared to the pure harmonic: $(1 / 144) / K D$ gives $\mathrm{H}^{0} \rightarrow 6.5, \mathrm{Z}^{0} \rightarrow 17.64, \mathrm{~W}^{ \pm} \rightarrow 61.74$. The overall range is comparable to quarks. 
Table 3. Massive bosons resonances $(*) \mathrm{MeV} / \mathrm{c}^{2}$

\begin{tabular}{lllllll}
\hline Particle & $\mathrm{P}$ & $\mathrm{N}$ & $\mathrm{K}$ & $\mathrm{NP}$ & Computed $(*)$ & Measured $(*)$ \\
\hline $\mathrm{W}^{ \pm}$ & 12 & 12 & -2 & 144 & $80,384.2$ & $80,385 \pm 15$ \\
$\mathrm{Z}^{0}$ & 12 & 12 & -7 & 144 & $91,187.67$ & $91,187.6 \pm 2.1$ \\
$\mathrm{H}^{0}$ & 12 & 12 & -19 & 144 & 126,355 & $125,500 \pm 400 \pm 400$ \\
\hline
\end{tabular}

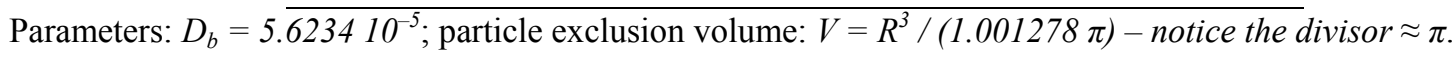

The distance $D_{b}$ was found by iterations starting with $D_{e} \mu / \alpha m_{e}$ (guessing an inversion between bosons and fermions) and then making first order adjustments in $\alpha$. Hence the coincidences:

$$
D_{b}=D_{e} \mu(1+7 \alpha / 3) / \alpha m_{e}=1.05612 \alpha^{2}
$$

The division of the exclusion volume by $1.001278 \pi$ was found empirically; it just works and we believe that no other simple solution does. It could come from a different density $(X)$ and then from a different field, but we will show that it is related to geometry. We also have $1.001278=1+24 \alpha^{2} \approx 1+(3 / 2) \alpha_{S} \alpha$; and then:

$$
V=R^{3} / \pi\left(1+24 \alpha^{2}\right) \approx R^{3} / \pi\left(1+(3 / 2) \alpha_{S} \alpha\right)
$$

But the most stunning coincidence is that we find $K$ in $\{-2,-7,-19\}$ in Table 3 , the same prime numbers as for quarks in Table 2, but column $N$. We also have $19-7=12=N$ and $P$ in Table 3 . Obviously, it means an inversion of geometry which is then our interpretation of the $\pi$ in (8). Moreover, as discussed in the previous section, a ratio 2 in quarks resonance changes the electric charge while 7 does not. But $K=2$ is associated to the $\mathrm{W}^{ \pm}$in Table 3, and then to quarks decay, while $K=7$ is associated to the neutral $\mathrm{Z}^{0}$; the coherence is stunning.

\section{Analysis of Resonance and Coincidences}

\subsection{Harmonics and Resonance Structure}

Equation (1) is well verified for all particles but we do not understand the resonances' geometry. Moreover the mass depends on the reverse of a volume $1 / R^{3}$ but the standard model spinors wave equations are based on the Compton wavelength $h / m c$. The Compton wavelength is proportional to $R^{3}$ but it is not easy to find a link between both quantities (except of course using the initial reasoning leading to (1); see Consiglio, 2012a); all we know is a proportionality relation $h / m c=k R^{3}$. It seems natural to analyze the resonance structure comparing the numbers in Tables $1,2,3$, and the different parameters $D$. It is essentially guesswork... but fortunately there is an unavoidable empirical $\pi$ in (8) that implies a difference in geometry between bosons and fermions.

Electrons obey the Dirac equation while bosons obey the Klein-Gordon equation; those equations do not have exactly the same physical significance, but sufficiently close for our purpose. Using $\AA=i \hbar / m c$ they are:

$$
\begin{aligned}
& \not \gamma^{\mu} \partial_{\mu} \Psi-\Psi=0 \\
& \AA^{2} \square \Psi-\Psi=0
\end{aligned}
$$

We have assumed a unique fundamental resonance $(N=P=1)$ and a unique field density $X$ for all particles. From (1) $\lambda$ is proportional to a 3 -volume, and it is used on equal grounds in the Dirac and Klein-Gordon equations. Since any function $\Psi$ verifying (9) will also verify (10) and since (9) addresses spin $1 / 2$ particles, then the resonances match a phase difference $4 \pi n$ in (9) and $2 \pi n$ in (10). But the volume is divided by $\pi$ for massive bosons; then it corresponds to a 4-volume divided by its radius in (9) and by its circumference in (10).

Whatever the space in which (1) works, the resonances are radial in Table 1, and circular in Table 3. Then in Table 2 it is a mix - P is radial and $N$ is circular since 2, 7 and 19 are inverted between Tables $2(N)$ and 3 (K).

Consider for electrons a fully radial double resonance. $N$ and $P$ are on the same axis as $K D_{e}$, which is then solely related to a repulsive force; there is nothing more to uncover in this resonance scheme alone.

For quarks, mixed resonance requires a phase lock between a circular $(N)$ and radial $(P)$ resonance; it implies a trivial relation between $\pi, 7,19$ and the constant $P=3$ in Table 2. We get:

$$
\begin{aligned}
7 \times 3 \pi & \approx 66 / 1.0004025 \\
19 \times 3 \pi & \approx 179 \times 1.0003954
\end{aligned}
$$

The discrepancies $\left(4 \cdot 10^{-4}\right)$ are small (and it can easily be checked that no other small prime number is so close to a solution of $3 \pi X=Y$, with $X$ and $Y$ integral numbers). 
Moreover:

$$
1.0003954 / 1.0004025 \approx 0.9999929
$$

They are the same at $7 \cdot 10^{-6}$, but of opposite signs. Finally, the numbers $7 \times 3 \pi$ and $19 \times 3 \pi$ are very close to circular harmonics of a radial resonance. The mixed resonance scheme holds but should be frequently broken, resulting in a conundrum of absorption/emission if compared to the perfect resonance of electrons - something we should probably expect from quarks as they exchange color charge while electrons exchange no charge. Now $(11-12-13)$ are trivial, but remember that the numbers 3,7 , and 19 were found empirically at the right places; the triviality is then a true confirmation that the empirical harmonics in Table 2 have geometric significance.

For massive bosons we should have a double circular or spherical resonance in $N P$, a situation similar to electrons except for $K D_{b}$ which must be part of the circular resonance path and is also necessarily radial - but with no radial resonance. Taking only the circular path into account (but keeping the constraint coming from the radius), $D_{b}$ should be a divisor of $\pi / 144$, a division that must hold with any $K$ in Table 3 . Since all those are primes numbers we can also use their product. Then in this simplified picture (that cannot hold) we should have:

$$
(\pi / 144) / D_{b}=2 \times 7 \times 19 \rightarrow \pi / 144=266 D_{b}
$$

Now $D_{b}$ is also radial and a volume depends on the cube of its radius. Then we must use $D_{b} \pi^{l / 3}$ on the right hand side; it gives a modified equation that is close to hold:

$$
\pi / 144=266 D_{b} \pi^{l / 3}
$$

But also, $\pi$ is multiplied by 1.001278 in (8); hence we must use the cube of this number on the left hand side, and reduce $\pi$ accordingly in $D_{b} \pi^{1 / 3}$; in this way we get comparable quantities, and we should have:

$$
(1.001278)^{3} \pi / 144=266 D_{b}(\pi / 1.001278)^{1 / 3}
$$

Actually, we find:

$$
(1.001278)^{3} \pi / 144=1.0000136 \times 266 D_{b}(\pi / 1.001278)^{1 / 3}
$$

The discrepancy is $1.4 \cdot 10^{-5}$. It shows that the resonance structure is coherent with empirical numbers, which definitely relate to some geometry and to the physics of mass, including $1.001278 \pi$.

Note also that updating $D_{b} \rightarrow 1.0000136 D_{b}$ to re-compute Table 3 has almost no effect on computed masses.

\subsection{A Single Field}

We have shown that our results are consistent and significant: All known masses are well approximated, and numbers and geometry are coherent. The ratios in coincidences are all based on simple equations in $\alpha$ or $\alpha_{S} \alpha$, and the prime numbers $2,3,7$, and 19 are coherent with geometry, as well as $1.001278 \pi$. It naturally suggests that a single phenomenon is at work giving all particles mass.

Nothing new one might say; of course, but the resonance structure depends on the symmetry or particles group and all Ds are defined from two coupling constants.

Therefore the SM Higgs field is sensitive to the type of charges carried by the particle.

- Firstly it is sensitive to all types of charges but in different manners - and then using some coincidences related to the Ds, we may understand how;

- Second, and of equal importance, the three forms of resonance can only be related to two types of charges and no more - because two forms are "pure" and one is mixed.

Let us use $X$ to denote the mass-giving field, $C$ for the quarks attractive force, and $E$ for electricity which is repulsive; putting charges and coincidences $(5-6-7)$ together with the resonances structure we get the following system:

$$
\begin{gathered}
U(1): X \times E \rightarrow \text { Radial }+ \text { radial } \rightarrow D_{e}=\alpha_{S} \alpha \approx 16 \alpha^{2} \\
S U(3): X \times(E+C) \rightarrow \text { Radial }+ \text { circular } \rightarrow D_{q}=\alpha_{S} \alpha(1+\alpha) \\
\text { Massive Bosons: } X \times X \rightarrow \text { Circular }+ \text { circular } \rightarrow D_{b}=1.05612 \alpha^{2}
\end{gathered}
$$

where all equations are coherent and have similar physical significances.

This system alone proves the existence of a single field below SM forces and fields, because E is electricity, $C$ is necessarily color charge, and $X$ is sensitive to both - there is a unique interaction. 


\subsection{A Physical Interpretation}

The system (15) can be understood in a coherent manner if we assume a fundamental difference between $X$ on the one hand, and $E, C$ on the other hand: The former is a current while the latter are static charges - and what we call a current is not simply a moving charge (like $J=e v$ ). Our interpretation is a single field breaking down and running as a function of mass and distance. In terms of running, (15) logically gives:

$$
\begin{gathered}
D_{e} / \alpha=\alpha_{S} \\
D_{q} / \alpha=\alpha_{S}(1+\alpha) \rightarrow \alpha_{S}(1+1.0276 \alpha) \\
D_{b} / \alpha=\alpha \rightarrow 1.0276 \alpha
\end{gathered}
$$

where the arrows represent the running, ranging in energy from the electron to the top quark and Higgs boson; in this picture only $\alpha$ runs. Note that $(1+\alpha)$ in (16.2) cannot be removed; since $K<0$ in Table 2 it relates to a distinct charge. The system can be solved for low masses if the $D s$ depend on potentials and two types of charges: $D$ is a priori a quantized distance, the trick is coherent with a potential.

For lower masses the respective charges and the force coefficients are:

$$
X=\alpha e ; C=\alpha e ; E E=X X: 1 ; X E=X C: \alpha_{S}
$$

where the notations are trivial. The charges $X$ and $C$ actually depend on the symmetry; with $\mathrm{U}(1), X=\alpha e / 2$.

The rationale for $(15-16) \rightarrow(17)$ is the following:

- $\quad C$ is color charge and $X$ and $C$ have the same type of charge (since 15.2 and 15.3 give the same coefficient $\alpha^{2}$ ); but $X$ is a current while $C$ is a static charge.

- Using the whole system (16), the force coefficients at short distance and low mass are:

$\circ \alpha_{S}$ between charges and currents,

- 1 between charges, $l$ between currents.

- Since $\alpha$ is the long range coupling of electrons with virtual photons in QED, the coupling is equivalent to a current of charges $\alpha e$ or two charges $\alpha e / 2$ - that we recognize in $(16.2-16.3)$. Then we identify QED virtual photons to $X$ or $X$-pairs currents, and we get:

$\circ$ All force coefficients at long range are 1 (including $\alpha_{S}$ ),

- A running of charge-currents interaction from long to short distance $\left(\alpha_{S}: 1 \rightarrow 0.11692\right)$.

- $\quad$ Equilibrium is reached from $X C=X X$ running positively at very short distance $(16.2-16.3)$.

- The running ( $\left.\alpha_{s}: 1 \rightarrow 0.11692\right)$ can also be seen as a density of charge separation (or field polarization), a breakdown of $X$-currents to more elementary currents under the influence of an electric charge.

- Massive bosons correspond to a resonance of the $X$-field alone giving (16.3), since $N P$ is constant.

The slope of the running has the shape of the Higgs potential; it is equivalent to a depletion volume of $X$ or $X$-pairs in agreement with (1), and it fits with the pressure field hypothesis leading to this equation.

Note that the geometry is coherent with a Higgs field appearing shortly after the big bang - when the density gets low enough and distances large enough for $X$-currents or $X$-pairs to form.

This is the minimal interpretation, it gives a single field, and it holds. Once understood the difference between static charges and currents, which are mandatory in an absorber theory, it comes naturally.

On qualitative grounds, we have $D_{q}>D_{e}>D_{b}$, and even $-K_{q} D_{q}>K_{e} D_{e}>-K_{b} D_{b}$ (where $K_{x}$ is any value of $K$ in the related table); hence the weaker the inner forces shaping the resonance structure, the higher the average $N P$ in a particle group, and the lesser the $K D$ - in agreement with the forces coefficients in (17).

From (16.2), we may have to update the large masses in Table 2 with $D_{q}=\alpha_{S} \alpha(1+1.0276 \alpha)$; the calculus gives the top mass $m_{t}=172.57 \mathrm{GeV} / \mathrm{c}^{2}$. Compare to $172.38 \mathrm{GeV} / \mathrm{c}^{2}$ in Table 2, it has no effect on the reasoning.

\section{Coherence With Magnetic Currents}

With respect to the SM Higgs field, $X$ must interact like a scalar field; it is charged but Lorentz invariant. It is then made of pseudo-scalar charges which, by Lorentz transformation, appear as an invariant current quantum $\boldsymbol{J}_{\boldsymbol{x}}$. For an electric charge, we have in standard notations:

$$
J_{e}=\rho_{e} \gamma v
$$

And then we should have similarly:

$$
\boldsymbol{J}_{\boldsymbol{x}}=\rho_{\boldsymbol{x}} \gamma \boldsymbol{v}
$$

Trivially, since $J_{x}$ is constant we get $\rho_{x}$ infinite for a particle at rest - then (19) is unphysical as it is. 
Thus nothing is at rest and there is a minimal velocity giving a maximal value to $\rho_{x}$. This velocity must be an invariant natural constant, it is then the speed of light; $X$ is tachyonic or light-speed. Then using $V=c^{2} / v$ and defining $\gamma_{S} V=\gamma c$, we can write:

$$
\boldsymbol{J}_{\boldsymbol{x}}=\rho_{\boldsymbol{x}} \gamma_{S} \boldsymbol{V}=\text { const.; } J_{x}=\rho_{x} \gamma c
$$

The $\gamma$ is attributed to the absorber/receiver time dilation, and then (20) can hold in two different manners which are not incompatible:

- As a charged tachyon, and we get $g V=$ const.

- As a light-speed constant charge; $\xi=$ const - which is doubtful in Minkowski space unless $\xi c=g V$.

The tachyon charge transformation in (20) was previously deduced in a different manner using the Bohr model and under the assumption that the electron mass is a magnetic current (Consiglio, 2013b); it gives a quantization condition opposite to Dirac's (1931), and agrees with the two interpretations above:

$$
g=Z \alpha e / 2 n ; g V=e c / 2
$$

with $n$ an integral number, and $Z e$ the nucleus charge in the Bohr model. Hence the charges in (17) are coherent with $Z=2, n=1$ or $Z=1, n=1$ for $g=\alpha e / 2$; the latter is of special interest as (21) gives $V=c / \alpha$, and then:

$$
\text { e } g V^{2} / c^{2}=h c / 2
$$

whose interest is obvious. But from (21), Equation (22) should be read: (e/2) $(e V / c)=h c / 2-$ as a tachyon current $(e / 2)$ seeing an electron $(e)$ with velocity $V$.

Last but not least, we recall that using the Lochak $(1995$ - 2007) monopoles theory, the predicted geometry can trivially be used to construct an $\mathrm{U}(3)$ or SU(3) basis (see Consiglio, 2012a), as it gives four types of monopoles combining charge sign $+/-$ and chirality $\mathrm{L} / \mathrm{R}$. It closes the loop with color charge as most elementary currents.

One could ask how we can get tachyons without mass, but it naturally reduces to mass without mass. Symmetry shows in our case that tachyons momentum is bradyons energy and conversely (Consiglio, 2013b); an elegant answer to the question: There is no mass, only currents, angular momentum, and action.

\section{On Fractional and Integral Charges}

Let us use, as a test, a 5D manifold of signature (+,-,-,-,-) for $(t, w, x, y, z)$, and then use $g V=e c / 2$ to get fractional charges from $v=d w / d t$. Using $V=c^{2} / v$ in (21) we have:

$$
e / 3=e v / 2 c ; 2 e / 3=e v^{\prime} / 2 c \rightarrow v=2 c / 3 ; v^{\prime}=4 c / 3
$$

It gives a velocity $c / 3$ in $w$, and tachyon velocities: $V=3 c / 2>c$ and $V^{\prime}=3 c / 4<c-$ but one is a bradyon, and then symmetry is broken in the direction $w$. Moreover, from (23) we can also use a simple addition of velocities. Assuming an unknown charge $\xi$ and a velocity $v$ in the direction $w$, one gets:

$$
e / 3=\xi(c+v) / c ; 2 e / 3=\xi(c-v) / c \rightarrow \xi=e / 2 ; v=c / 3
$$

Equations like (23) and (24) do not hold together for other fractions, except for $1 / 2$ and $v=c$.

We also get an integral charge mixing two currents of opposite charges and directions. On this basis, and assuming a few simple rules, it is quite easy to build all SM particles and symmetries out of combinations of currents. In this picture, the currents $e / 3$ and $2 e / 3$ define particles inner circulation of Equation (1), while charged tachyons and light-speed neutral currents correspond to the absorption/emission process of distinct symmetries (and interestingly, it gives no gravitons). Eventually, we get $\mathrm{U}(3)$ broken in the $w$ direction (or a combination of two $\mathrm{U}(3)$, one broken or of three $\mathrm{U}(1)$, one broken).

This direction corresponds to a $4^{\text {th }}$ space dimension, but it might not be needed; since one of the fractional charges is a tachyon and the other a bradyon, then the symmetry of space-time is broken ("before any interaction") and it might be simply $w=t$, this dimension appearing simultaneously as space and time (recall also that a tachyon sees $3 D$ space as 3 dimensions of time and $1 D$ time as a dimension of space). The picture is far from complete, but so logical, minimal, and elegant that it needs to be mentioned.

\section{Conclusions}

The logic in this paper is coherent with the observed mass spectrum and consistent in resonance numbers and geometry. No better confirmation of the validity of Equation (1) can exist. We showed that the mass spectrum of elementary particles is natural and that a single field is necessary and sufficient to explain it. The field interpretation agrees, directly from (1) and the mass spectrum, with the electromagnetic and strong forces coupling constants, and with the shape of the Higgs potential. It is below SM forces and fields. 
An excel spreadsheet is provided as supplementary material with computations related to the resonance tables and coincidences.

\section{Addendum 1}

Without any change to our conclusions, we can write (15.3) in a different way, and with very good precision:

$$
\text { Massive Bosons: } ? \times ? \rightarrow \text { Circular }+ \text { circular } \rightarrow D_{b}=\alpha^{2}\left(1+\alpha_{S} / 2-\alpha_{S}^{2} / 6\right)
$$

Then (16) becomes simpler since $\alpha$ is not running. But the interpretation requires X-charges and $\mathrm{C}$-charges at work in (15.3a) to get the first two coefficients. $\left(\alpha^{2} \rightarrow X \times X\right.$ or $C \times C$, and $\left.\alpha^{2} \alpha_{S} / 2 \rightarrow X \times C\right)$.

\section{Addendum 2 - Comparing Resonance Quarks Masses With Lattice QCD Results}

Let us compare the quarks masses computed in Table 2 with known estimate from lattice QCD given by Davies $\&$ al (2010) - the exercise is of particular interest since one can hardly find more opposite or complimentary approaches. Lattice QCD simulation gives:

- $\quad m_{u}=2.01 \pm 0.14{\mathrm{MeV} / \mathrm{c}^{2},}^{2}$

- $\quad m_{d}=4.79 \pm 0.16 \mathrm{MeV} / \mathrm{c}^{2}$,

- $\quad u d$ average $m_{l}=3.40 \pm 0.07 \mathrm{MeV} / \mathrm{c}^{2}$.

- $\quad m_{c} / m_{s}=11.85 \pm 0.16$.

We find in Table 2 differences of $-3.9 \%,+4.4 \%$ for individual masses ( $u, d$, respectively), and a nice agreement in average with $m_{l}=3.47 \mathrm{MeV} / \mathrm{c}^{2}$. Interestingly, the differences are opposite and almost complimentary, in a manner similar to the resonance breaches discussed in section 3.1.

The $m_{c} / m_{s}$ ratio is also in great agreement as we get $m_{c} / m_{s}=11.797$ from Table 2 .

\section{Addendum 3 - A Better Link With QED Concepts}

Equation (1) was initially deduced from the assumption of a pressure field of "elementary momentum carriers", so called Universons. We then departed from the "momentum only" assumption, and used some charges, essentially because "momentum only" is quite limited in terms of interaction and symmetry. In Universons theory, the "particle picture" is the following:

- A flux $\Phi$ exists of invariant elementary momentum carriers of energy $E_{U}$.

- Particles have a (particle-type dependent) cross-section $S$ with the flux.

- A momentum carrier is absorbed/retained and reemitted after a particle-independent time $\tau$.

The energy of a particle is $E=F(\Phi, S)$, since $E_{U}$ and $\tau$ are constants. In this view an electron's electric charge has no intrinsic energy, all its energy is made of absorbed Universons; the concept is physically irrelevant because it assumes that something exists with no energy - an acceptable modification of this equation is:

$$
E=F(\Phi, S)+\mu c^{2}
$$

Where $\mu$ is the same as in Equation (1), this is what we call the electron proper energy or "naked mass" - but it is not QED self-interaction which is $F(\Phi, S)$. Thus:

- We first say that QED models this function $F$ with virtual photon loops, then $F=E=$ self-interaction and in this interpretation there is no need for the mass $\mu$.

- Second, we say that this function $F$ is the absorber and that obviously, the absorption/emission as well as the retention processes addresses the quantum of action $h$ and of angular momentum $t / 2$. Equation (1), in its origin, also addresses particle-wave duality.

Therefore, a quantized absorber is relevant; if a quantum of absorption exists, since the electric charge does not depend on the electron's energy (or muon, or tau), there is necessarily an energy associated to this charge. Then the absorption is $F(\Phi, S)=(m-\mu) c^{2}=$ absorption/emission, and $\mu$ is a "naked mass". In this way, our comparison with Bethe's results is relevant.

On this basis let us look at the magnetic current interaction; using the minimal Dirac charge $g_{D}=e / 2 \alpha$, we have:

$$
E=h v=2 \pi e\left(2 g_{D}\right) v / c
$$

which, in this form, addresses a quantized magnetic current $2 g_{D} v$. It relates to a current, not to a pair of charges. Assume that an electron proper energy $(\mu)$ depends on the square of its charge: $e^{2}$ gives the mass $\mu$, and then we can define a constant $L$ that verifies:

$$
L e^{2}=\mu c^{2}
$$


From (A1), the function $F$ is given by the electron charge interaction with a (hypothetical) pair of Dirac charges, which corresponds to retention. Using the conservation of energy, it should be equal to the proper energy of two hypothetical Dirac charges; we can then define two other constants $L$ ' and $L$ " verifying:

$$
\left(L, \text { e } 2 g_{D}=\right) 2 L^{\prime} g_{D}^{2}=\left(m_{e}-\mu\right) c^{2}
$$

Then from (A2 - A3), and replacing $g_{D}$, we get:

$$
\mu / L=2 \alpha^{2}\left(m_{e}-\mu\right) / L,
$$

With a unique field, the ratio $L / L^{\prime}$ must be related solely to geometry and then it cannot be arbitrarily large or small; thus Equation (A4) is in formal agreement with the coincidence (3), from which we get:

$$
L / L^{\prime}=2 \pi / \sqrt{ } 2
$$

Hence the coincidence (3) can be related to a unique field, to geometry, and to the Dirac condition.

\section{References}

ATLAS Collaboration. (2013). Combined measurements of the mass and signal strength of the Higgs-like boson with the ATLAS detector using up to $25 \mathrm{fb}-1$ of proton-proton collision data. Retrieved from http://cds.cern.ch/record/1523727

Bethe, H. A. (1947). The electromagnetic shift of energy levels. Phys. Rev., 72, 339. http://dx.doi.org/10.1103/PhysRev.72.339

Consiglio, J. (2012a). On particles mass and the Universons hypothesis. Applied Physics Research, 4(2), 144-158. http://dx.doi.org/10.5539/apr.v4n2p144

Consiglio, J. (2012b). Relativity, weak bosons mass and magnetic charges. Applied Physics Research, 4(3), 100-114. http://dx.doi.org/10.5539/apr.v4n3p100

Consiglio, J. (2013a). Quantized charges and currents in the electromagnetic field. Applied Physics Research, 5(1), 126-134. http://dx.doi.org/10.5539/apr.v5n1p126

Consiglio, J. (2013b). Minkowski space and Tachyons velocity. Applied Physics Research, 5(6), 1-14. http://dx.doi.org/10.5539/apr.v5n6p1

Davies, C. T. H., McNeile, C., Wong, K. Y., Follana, E., Horgan, R., Hornboste, K., \& Trottier, H. (2010). Precise Charm to Strange Mass Ratio and Light Quark Masses from Full Lattice QCD. Physical Review Letters, 104, 132003. http://dx.doi.org/10.1103/PhysRevLett.104.132003

Dirac, P. A. M. (1931). Quantized singularities in the Electromagnetic Field, Proc. Roy. Soc., A, 133, 60.

Dirac, P. A. M. (1948). The Theory of Magnetic Poles. Phys. Rev., 74, 817.

Lochak, G. (1995). The Symmetry between Electricity and Magnetism. Advanced Electromagnetism. World Scientific, Singapore. Retrieved from http://aflb.ensmp.fr/MEMOS/GLmonopole/MONOPOLE.pdf

Lochak, G. (2007). The symmetry between Electricity and Magnetism and the equation of a leptonic Monopole Fondation Louis de Broglie. Retrieved from http://arxiv.org/abs/0801.2752

Mohr, P. J. (2012). CODATA Recommended Values of the Fundamental Physical Constants: 2010. Retrieved from http://arxiv.org/pdf/1203.5425.pdf

Recami, E. (1976). Magnetic Monopoles and Tachyons in Special Relativity. Physics Letters B, 62(1), 41-43. http://dx.doi.org/10.1016/0370-2693(76)90042-3

\section{Copyrights}

Copyright for this article is retained by the author(s), with first publication rights granted to the journal.

This is an open-access article distributed under the terms and conditions of the Creative Commons Attribution license (http://creativecommons.org/licenses/by/3.0/). 